\title{
Design of 3D-printed Cable Driven Humanoid Hand Based on Bidirectional Elastomeric Passive Transmission
}

\author{
Teru Chen ${ }^{1}$, Xingwei Zhao ${ }^{1}$, Guocai Ma ${ }^{2}$, Bo Tao ${ }^{1^{*}}$ (I) and Zhouping Yin ${ }^{1}$
}

\begin{abstract}
Motion control of the human hand is the most complex part of the human body. It has always been a challenge for a good balance between the cost, weight, responding speed, grasping force, finger extension, and dexterity of prosthetic hand. To solve these issues, a 3D-printed cable driven humanoid hand based on bidirectional elastomeric passive transmission (BEPT) is designed in this paper. A semi-static model of BEPT is investigated based on energy conservation law to analyze the mechanical properties of BEPT and a dynamical simulation of finger grasping is conducted. For a good imitation of human hand and an excellent grasping performance, specific BEPT is selected according to human finger grasping experiments. The advantage of BEPT based humanoid hand is that a good balance between the price and performance of the humanoid hand is achieved. Experiments proved that the designed prosthetic hand's single fingertip force can reach $33 \mathrm{~N}$ and the fastest fingertip grasping speed realized $0.6 \mathrm{~s} / 180^{\circ}$. It also has a good force compliance effect with only 430g's weight. It can not only grab fragile objects like raw eggs and paper cup, but also achieve strong grasping force to damage metal cans. This humanoid hand has considerable application prospects in artificial prosthesis, human-computer interaction, and robot operation.
\end{abstract}

Keywords: Humanoid hand, Bidirectional elastomeric passive transmission, Artificial prosthesis

\section{Introduction}

Through millions of years of evolution, human have a facile and strong hand that no other spiritual claw animal possess [1-3]. The hand is seen as the extension of the brain. Motion control of the human hand is the most complex part of the human body $[4,5]$. From ancient fixtures to modern prosthetic hand, it has been a challenge for hundreds of years to fabricate a humanoid hand that can replace the human hand [6]. Losing hands can greatly affect a person's quality of life. According to statistics, nowadays there are more than 20 million physically disabled people in China, accounting for $2 \%$ of the total population. Therefore, artificial prosthetic hands play

\footnotetext{
*Correspondence: taobo@hust.edu.cn

${ }^{1}$ State Key Laboratory of Digital Manufacturing Equipment

and Technology, Huazhong University of Science and Technology,

Wuhan 430074, China

Full list of author information is available at the end of the article
}

an important role in improving the lives of people with disabilities and in medical rehabilitation $[7,8]$. However, current famous humanoid hand in the world like iLimb (Touch Bionics Inc.), Vicent Hand (Vicent Inc.), and Bebionic hand (Otto Bock Inc.) are still far away from this goal [9].

Limited by narrow space, motor price, kind of transmission, and manufacture process, it has always been a problem to reach a good balance between the cost, weight, responding speed, grasping force, finger extension, and dexterity of prosthetic hand [10]. According to the user studies, $90 \%$ of patients feel their myoelectric prostheses too slow and 79\% feel too heavy [11]. Humanoid hand with good performance always has an expensive price, such as BeBionic Hand (Ottobock; \$11000) and the iLimb (Touch Bionics Inc.; $\$ 18000$ ), which most of patients can't afford. On the contrary, cheap value hands cannot provide a good performance guarantee. In 2004, 
HIT designed a novel dexterous robot hand with a fingertip force about $10 \mathrm{~N}$, far away from human's ability [12]. In 2010, Lee at al. designed a prosthetic hand has fingertip force of $20 \mathrm{~N}$, but this hand's weight reached up to 721 g [13]. In 2018, a Multi-Grip Patterns Prosthetic Hand with Single Actuator was manufactured by Panipat Wattanasiri. This hand has a great grasp force of $34.5 \mathrm{~N}$ but the hand closing time is 1.4-1.7 s [14], still can't realize a great performance balance.

The appearance of load-sensitive continuously variable transmission (CVT) [15] proposed a possible solution to this problem. It could adjust the motor's effective gear ratio according to the cable's tension. There are many kinds of CVTs invented in last few years, in 2012, a robot finger design using a dual-mode twisting mechanism to achieve high-speed motion and large grasping force [16] was proposed. In 2014, a passive, origami-inspired CVT [17] is published. However, current CVTs are limited by volume requirement, work modes, craftsmanship and other restrictions, can't support a good practical effect.

Due to the excessive degrees of freedom of the human hand, scientists usually use under-actuated mechanisms when designing artificial prosthetic hands. They ignore some unimportant degrees of freedom and try to preserve the characteristics of human hand as much as possible [18-20]. In 2016, Xiong et al designed an anthropomorphic hand for replicating human grasping functions. They used four motors to achieve $91 \%$ of the manual grasping mode [21-23], but their hand's fingertips force are still relatively poor. At the same time, the extension mechanism of the robot finger has been a problem for a long time. In many papers, humanoid hands were invented without extension mechanism or with heavy extension mechanism [24, 25]. Currently, a famous robot finger extension mechanism is torque spring [26]. But torque spring will influence the performance of robot fingers such as responding speed and grasping force. In Ref. [27], an Artificial Muscle Actuated Finger towards Biomimetic Prosthetic Hands is designed, the author designed an extensor tendon to extend the finger. But his design has too many degrees of freedom, resulting in not very good results.

Committed to solving the problems mentioned above, we proposed bidirectional elastomeric passive transmission (BEPT) and corresponding 3D-printed cable driven humanoid hand. The main contributions of this article are listed as follows:

1) A new kind of transmission mechanism: Bidirectional elastomeric passive transmission (BEPT) was designed in this paper. Through the employment of the flexible fence and the extension wheel, the force- speed compliance, flexion, and extension of the finger can be realized simultaneously under a motor drive.

2) The kinematics and dynamic transmission process of BEPT were investigated. The simulation and results of experiments confirmed each other, prove the correctness of the analysis.

3) Based on BEPT, a humanoid hand was designed and manufactured through a DLP 3D-printer. With a price less than 50 dollars and equal size to a human hand, the humanoid hand has a $33 \mathrm{~N}$ largest fingertip grasping force and $0.6 \mathrm{~s} / 180^{\circ}$ fastest fingertip speed, which are almost equal to humans. At the same time, the weight of the $3 \mathrm{D}$-printed humanoid hand is less than $430 \mathrm{~g}$ (adult man: 400-500 g). Through the application of BEPT, the designed humanoid hand can reach a great balance between the cost, weight, responding speed, grasping force, finger extension and dexterity.

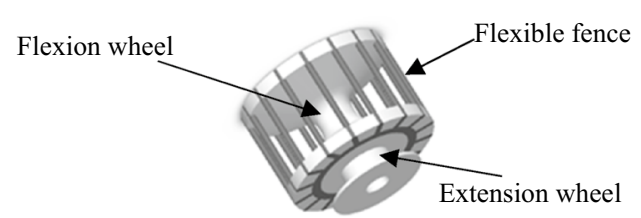

(a)

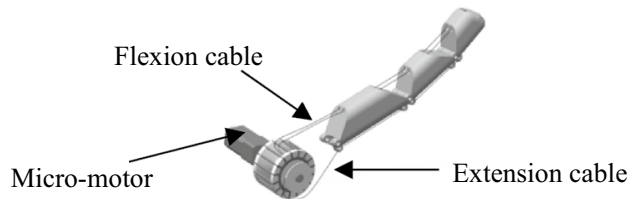

(b)

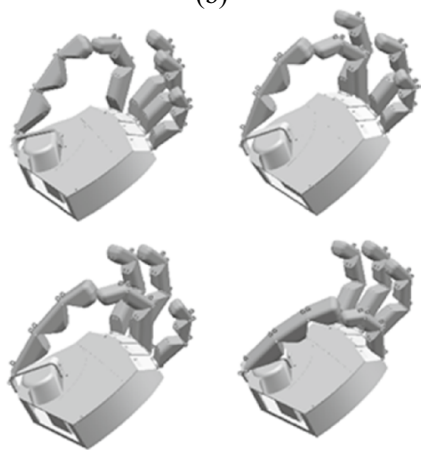

(c)

Figure 1 (a) Structure of BEPT, (b) Transmission process, (c) CAD-design of humanoid hand 


\section{Design of BEPT and Humanoid Hand}

\subsection{BEPT Design and Manufacture}

Figure 1(a) shows the main structure of bidirectional elastomeric passive transmission (BEPT), the BEPT is composed of two parts: the flexion wheel and the extension wheel. The flexion wheel consists of the rigid core and peripheral flexible fence. The BEPT is inspired by the work of O'Brien [28], the innovation of our work is that an extension wheel is added to EPT and the number of flexible fences can be easily increased or decreased to change the dynamic response of each finger. Two groups of cables are applied to complete the flexion and extension movement of fingers. Winding on the outside of flexion wheel, flexion cable takes the role of controlling grasping movement of the finger. While extension cable winds on the extension wheel, responsible for the extension movement of the finger. The schematic diagram of the transmission process can be seen in Figure 1(b).

A DLP 3D-printer is utilized for the manufacture of BEPT. The rigid part of BEPT (including the extension wheel and core of the flexion wheel) is $3 \mathrm{D}$ printed by common photosensitive resin. There are two materials for flexible fence fabricating: industrial flexible polyurethane and elastic resin (LCD-E, SparkMaker Corporation). Both of these two materials have approximate physical properties to each other. The front is selected to fabricate flexible fence. Flexible fence and rigid part are bonded together by superglue and placed in the sunlight for $2 \mathrm{~h}$ for curing.

\subsection{Design of Humanoid Hand}

Based on the design of BEPT and the conception of bidirectional transmission. A humanoid hand is designed and the image is shown in Figure 1(c). The shape of humanoid hand is designed imitating the profile of human hand, including a big and strong thumb, long middle finger and short little finger. Five micro-motors and BEPTs can be placed in the palm for a compact structure. The size of the humanoid hand is equal to an adult male hand.

Having advantages like fewer drive motors and good compliance, tendon driven system is commonly used in humanoid hand field. The tendon driven system usually adopts an under-driving driving mode, which can reduce the number of driving motors, and is beneficial to the overall weight loss of the prosthetic hand. The hand designed in this paper adopted a tendon driven system. On the front and back of the finger, guided holes are set to cross corresponding cables.

Human hand has excellent operational ability because of a flexible and strong thumb. For better operational performance, a two DOF thumb is designed in our humanoid hand. We added a miniature steering gear to add a degree of freedom of rotation to the thumb, which can make the movement of the thumb more flexible. With both rotation and opening-closing freedoms, the thumb can easily make a collision with other 4 fingers to form diverse grasping patterns [29].

\section{Performance Analysis of Humanoid Hand}

\subsection{Kinematic Analysis for Radius Ratio}

During the finger's flexion and extension, it is essential to maintain the slack condition of the non-shrinking cable. When the finger is grasping, flexion cable is stretched by the flexion wheel and extension cable is loosed by the extension wheel, so the length of extension wheel loosed must be longer than the finger joint needed. Only in this way can we avoid the impact on the grasping performance of the fingers and the damage to the fingers and the motor during grasp. The same is true when the finger reverts.

Taking a single finger joint as an example in Figure 2(a), it is assumed that the BEPT's $R_{o}$ is not shrunk during grasping, the rotation angle of BEPT is $\theta$, we can get the length of the flexion cable is stretched:

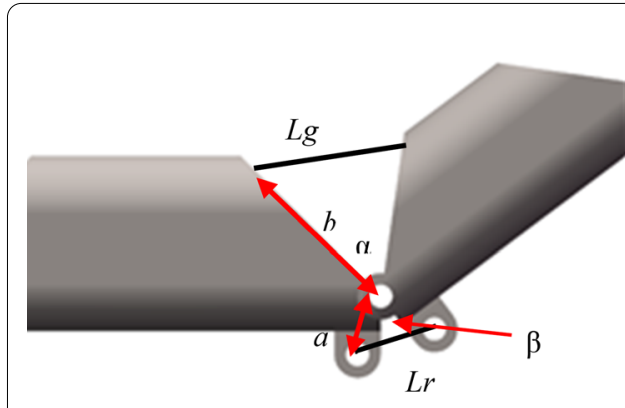

(a)

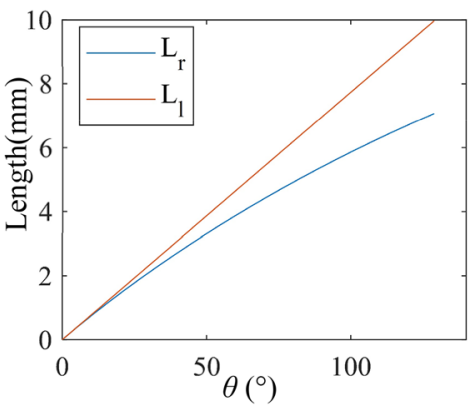

(b)

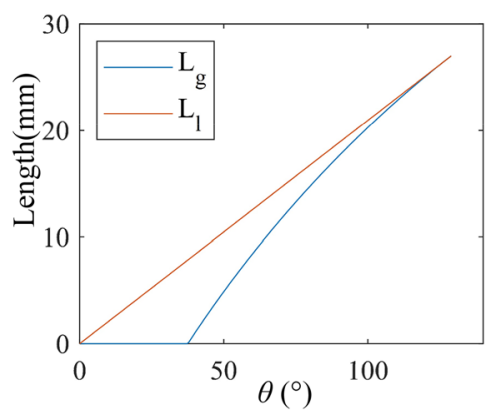

(c)

Figure 2 (a) Finger joint in the grasp process, (b) Length change of $L_{l}$ and $L_{r}$ during flexion, (c) Length change of $L_{l}$ and $L g$ during extension 


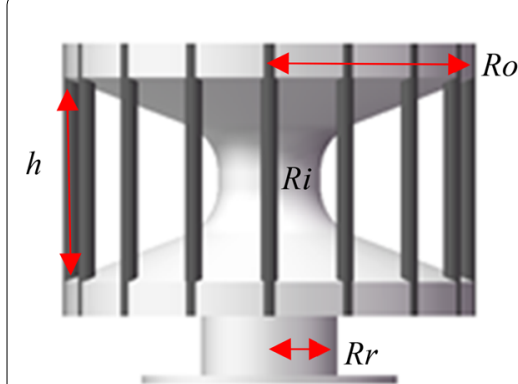

(a)

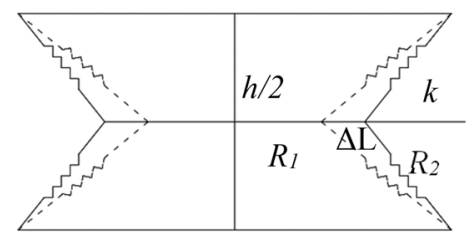

(b)

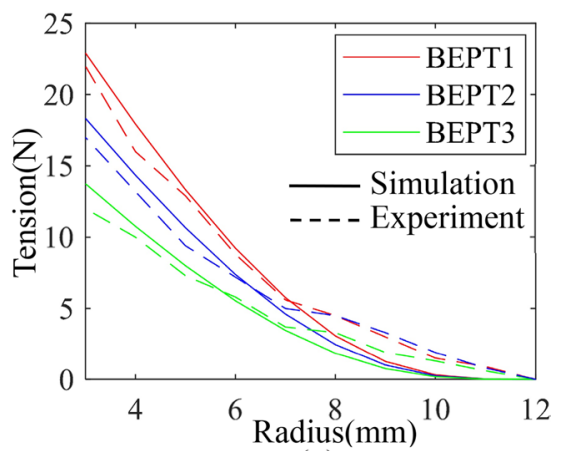

(c)

Figure 3 (a) Main geometric parameters of BEPT, (b) Static spring-cable system, (c) Comparison between simulation and experiment of BEPT1,2,3

$$
\Delta L=R_{o} \cdot \theta .
$$

Then real-time angle $\alpha$ can be written as:

$$
\alpha=\cos ^{-1}\left[\frac{2 b^{2}-(L-\Delta L)^{2}}{2 b^{2}}\right],
$$

where $L$ is the length of flexion cable in the condition of the finger joint is fully extended and $b$ is the length between the joint and the guided hole. In our design, we set the maximum of $\alpha$ is $135^{\circ}$, so the value of $\beta$ is:

$$
\beta=135^{\circ}-\alpha .
$$

$a$ is the length between the joint and the extension guided hole. The length that extension cable needed for flexion can be written as:

$$
L_{r}=\left(2 a^{2}-2 a^{2} \cos \beta\right) .
$$

From Eqs. (1)-(4) we can get that $L_{r}$ is a function of $\theta$, and it must be satisfied that the length of extension wheel loosed $L_{l}$ must be longer that the extension cable needed $L_{r}$

$$
L_{l}=R_{r} \cdot \theta>L_{r} .
$$

So the suitable minimum value of $R_{r}$ :

$$
R_{r}=\max \left(\frac{\partial L_{r}}{\partial \theta}\right)
$$

In the design of humanoid hand, the length of $b$ and $a$ is $19.1 \mathrm{~mm}$ and $5 \mathrm{~mm}$ respectively. Through simulation we can get that the change of $L_{l}$ and $L_{r}$ during grasping in Figure 2(b). $L_{l}$ is always longer than $L_{r}$, it is proved that the grasping performance is not be effected. According to similar process, the simulation of the process of extension also shows the same result in Figure 2(c). Where $L_{g}$
Table 1 Main Parameters of each kind of BEPT

\begin{tabular}{ll}
\hline Number & Variables \\
\hline BEPT1 & $R o=12 \mathrm{~mm}, R i=3 \mathrm{~mm}, h=16 \mathrm{~mm}, N=20$ \\
BEPT2 & $R o=12 \mathrm{~mm}, R i=3 \mathrm{~mm}, h=16 \mathrm{~mm}, N=16$ \\
BEPT3 & $R o=12 \mathrm{~mm}, R i=3 \mathrm{~mm}, h=16 \mathrm{~mm}, N=12$ \\
BEPT4 & $R o=12 \mathrm{~mm}, R i=4 \mathrm{~mm}, h=16 \mathrm{~mm}, N=20$ \\
BEPT5 & $R o=12 \mathrm{~mm}, R i=5 \mathrm{~mm}, h=16 \mathrm{~mm}, N=20$ \\
\hline
\end{tabular}

Table 2 Different BEPT for Specific Finger

\begin{tabular}{ll}
\hline Finger & BEPT \\
\hline Thumb & BEPT1 \\
Index finger & BEPT1 \\
Middle finger & BEPT1 \\
Ring finger & BEPT4 \\
Little finger & BEPT5 \\
\hline
\end{tabular}

represents the length of the cable required to complete the extension of the prosthetic hand, and $L_{l}$ represents the length of the cable actually released during this process. $L_{l}$ is always more than $L_{g}$ and $L_{r}$, which means that the prosthetic hand can smoothly complete the grasping and extension actions.

\subsection{Performance Analysis of BEPT}

In this section, the semi-static mechanical model of BEPT is proposed. The main geometric parameters of BEPT marked in Figure 3(a): $R_{o}$ is the outside radius of flexion wheel, $R_{i}$ is the smallest radius of the rigid core, $R_{r}$ is the radius of extension wheel, $N$ is the number of flexible fences, $h$ is the height of flexible fence and $k$ is the tensile stiffness of a single fence strut. $R_{o}$ decides the biggest grasping speed of the finger, the larger of $R_{o}$, the faster grasping speed can be achieved. While $R_{i}$ determines the largest grasping force of fingertip, $N$ and $k$ decide the transmission characteristics of BEPT. 
To research the relationship between $N, k$, and cable tension $T, 5$ different BEPT are picked out for performance analysis. The main parameters of different BEPTs are shown in Table 1.

The energy conservation law is adopted to analyze the relationship between transmission radius $R$ and the tendon tension $T$. Due to the low weight of BEPT, the kinetic energy of BEPT can be ignored. By further neglecting the friction between the cable and the fence strut, the whole transmission system can be predigested to a static spring-cable system.

For a differential radius change $\Delta R=R_{2}-R_{1}$, from Pythagorean theorem and Hooke's law, the elastic potential energy change of springs can be expressed as:

$$
\begin{aligned}
& \Delta p=2 N \times \frac{1}{2} k\left[\sqrt{\left(R o-R_{1}\right)^{2}+\left(\frac{h}{2}\right)^{2}}\right. \\
& \left.-\sqrt{\left(R o-R_{2}\right)^{2}+\left(\frac{h}{2}\right)^{2}}\right]^{2} .
\end{aligned}
$$

Corresponding elongation (movement amount) of the scope:

$$
\Delta L=2 \pi R_{2}-2 \pi R_{1}=2 \pi \Delta R .
$$

With regard to a static condition, the cable tension $T$ :

$$
\begin{aligned}
& T=\frac{\Delta p}{\Delta L}= \\
& \frac{N k\left[\sqrt{\left(R_{o}-R_{1}\right)^{2}+\left(\frac{h}{2}\right)^{2}}-\sqrt{\left(R_{o}-R_{2}\right)^{2}+\left(\frac{h}{2}\right)^{2}}\right]^{2}}{2 \pi\left(R_{2}-R_{1}\right)} .
\end{aligned}
$$

Experiments are conducted to verify the theoretical derivation. Because the difference between BEPT3, BEPT4, and BEPT5 is only $R_{i}$, they have similar transmission process except for a different largest cable tension. So we only verified the relationship between $R$ and $T$ of BEPT1, BEPT2, and BEPT3.

The results of simulation and experiments are shown in Figure 3(c). The solid lines present the consequence of simulations and the dotted lines indicate experiments. Due to the nonlinear characteristic of the polyurethane, we can get that in the early stage of BEPT contraction, the cable tension of experiment is bigger than simulation and in the late stage it takes place an opposite result. Overall, the results of the experiments and simulation are relatively consistent. It's reasonable to define and adjust the performance of BEPT through the result of simulations. For instance, if we want to improve the stiffness of BEPT, we can increase the number of the flexible fences. If we want to add the largest force of fingertip, we can decrease the smallest radius of rigid core $R_{i}$.

\subsection{Dynamics Simulation of Finger Grasping}

In this section, in order to understand how the grasping force changes when the finger is grasping an object, a simulation of dynamics of finger grasping is conducted. As Figure 4(a) and Figure 4(b) shows, two types of simulation are carried out for a comparison, one is grasping a rigid object and another is elastic object.

Firstly, a classical mechanical transmission dynamic equation is written as:

$$
T_{M}-T_{L}=\frac{G D^{2}}{375} \frac{\mathrm{d} n}{\mathrm{~d} t},
$$

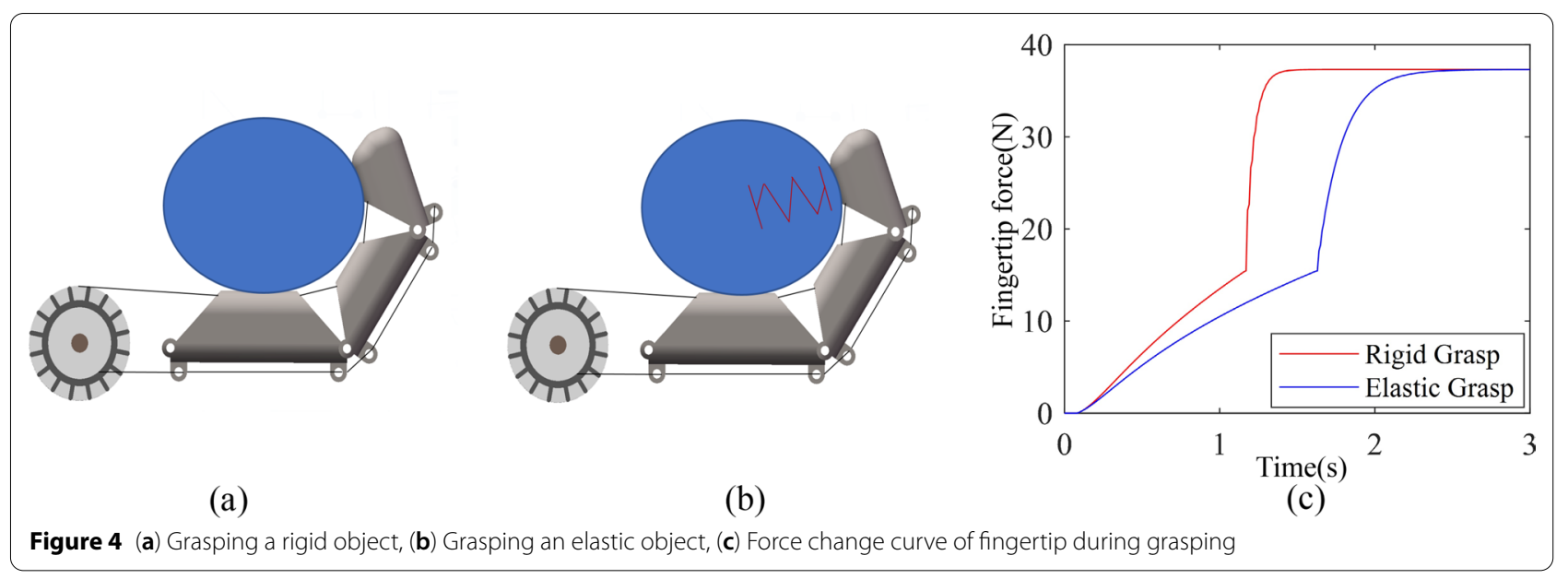


where $T_{M}$ is the torque of motor and $T_{L}$ is load Torque. $G$ and $D$ is constants about motor configuration. The $n$ is motor's rotating speed. At the meantime, the mechanical characteristics of the motor is:

$$
n=\frac{U}{K_{t} \Phi}-\frac{R_{a}}{K_{e} \Phi} T_{M} .
$$

Among them, $n$ is a function of $T_{M}, K_{t}, \Phi, K_{e}$, and $R_{a}$ are constants. In this paper, we use the GA12-N20 geared motor as the drive motor with a drive voltage of $12 \mathrm{~V}$ and a reduction ratio of 1:200. According to the data given by the merchant, we can get the mechanical characteristic curve of the motor:

$$
T_{M}=-16.037 n+248.21 .
$$

where the unit of $T_{M}$ and $n$ is N.mm and rad/s. Simultaneously, we should know the relationship between the BEPT's tension $T$ and real-time transmission radius $R_{T}$, here we used previous semi-static model of BEPT1 in Section 3.2 to solve the relation between $T$ and $R_{T}$. In the process of rotation, the initial rotation speed $n_{0}$ of the motor is given, the amount of rope involved and radius reduction of $\mathrm{BEPT}$ can be obtained as:

$$
\begin{aligned}
& \Delta l=n_{0} \cdot R_{T}, \\
& \Delta R=\frac{\Delta l}{2 \pi} .
\end{aligned}
$$

According to $\Delta R$ we can know the real-time transmission radius $R_{T}$ and tension $T$ of BEPT. During rotation, $T_{L}$ is a function of tension $T$ :

$$
T_{L}=T \cdot R_{T} .
$$

Through Eqs. (10) and (12) we can know the motor torque and motor shaft acceleration, then solve the whole process of gripping force change.

As for the process of grasping an elastic object, there only one thing need to be noticed is that due to the elasticity of the objective will cause the fingers to move additionally, which will bring extra cable length to $\mathrm{BEPT}$, resulting in a reduction in the motor acceleration. Assuming $K_{o}$ is the stiffness of the target object, the relationship between the extra cable length $\Delta l_{e}$ and the force $F_{t}$ at the fingertip is:

$$
\begin{aligned}
& \Delta l_{e}=F_{t} / K_{o}, \\
& \Delta l=n_{0} \cdot R_{T}-\Delta l_{e} .
\end{aligned}
$$

At each moment, the extra cable length needs to be subtracted when calculating the amount of cable involved. Other solving steps is similar to the process of grasping a rigid object.

The results of the simulation are shown in Figure 4(c), the red line shows the force change curve when grabbing a rigid body and the blue line shows the elastic object. It can be seen from the picture that the change of force is divided into two stages. In the first stage, BEPT does not fully contract, and the force increases relatively slowly. In the second stage, BEPT completely contracts, the motor stalls and the force increases rapidly. It is worth noting that there is an inflection point between the two stages. The position of the inflection point is determined by the maximum tension of the BEPT. We can adjust the inflection point by adjusting the number of flexible fences. Due to the elasticity of the elastic objective, the force changes of grasping an elastic objective is slower than the rigid object, but the final gripping force is equal. Since our simulation did not consider the friction of the motor reduction gear set and the rope as well as other factors that cause energy loss, the actual gripping force will be smaller than the simulation. But our design goal is achieved, that is, by adopting BEPT, we can achieve good results both in finger grasping speed and grasping force.

\subsection{Finger Heterogeneous Design}

Considering that human hand has different five fingers and different fingers have their own unique roles to make a stable and strong grasp, it's necessary that different BEPT should be chosen to specific finger for a better imitation to human hand. Firstly, a test need to be done to find the grasping character of specific fingers. In this test, different fingers are required to grasp a force sensor and a corresponding fingertip grasping force-time curve is recorded. To reduce the influence of unrelated factors, the force sensor is grasped by only the testing finger without other finger's help. Test results are shown in Figure 5(a). Through this test we can obtain that the strongest finger of human hand is thumb with around 20 $\mathrm{N}$ fingertip force. Index finger, middle finger, ring finger and little finger decrease in fingertip force in turn. Little finger has the smallest force about $12 \mathrm{~N}$.

For the purpose of analyzing if the force distribution form of human fingers has advantages, a simple simulation is carried out in the situation of grasping a virtual sphere. In simulation, we set up two different humanoid hand that one's 5 fingers have a force distribution like human fingers and the other's 5 fingers have equal grasping force. The homogeneous and heterogeneous hand were commanded to grab a sphere with different diameter. The contact constraints between 


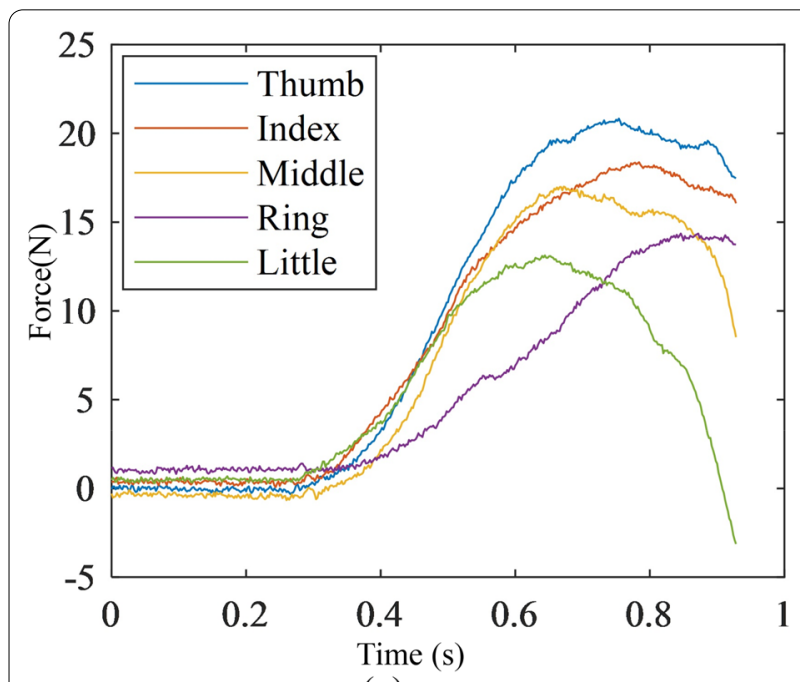

(a)

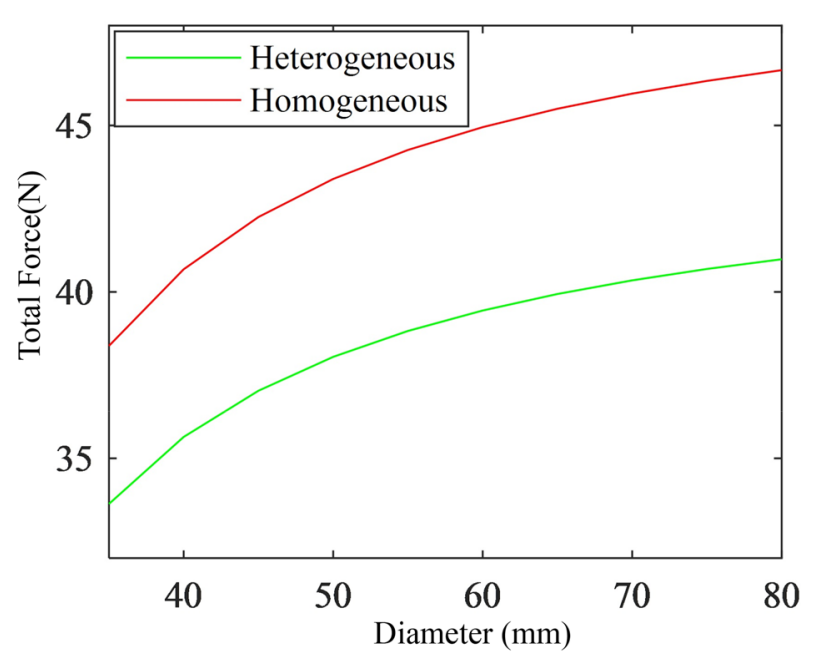

(b)

Figure 5 (a) Grasping curve of different human fingers, (b) Comparison of homogeneous and heterogeneous grasping

the fingertips and the objects can be modeled as a nofriction contact. The contact wrench of each point is expressed in the local coordinate system as:

$$
F_{i}^{C}=\left[\begin{array}{l}
f_{i}^{c} \\
\tau_{i}^{c}
\end{array}\right], f_{i}^{c}=\left[\begin{array}{l}
0 \\
0 \\
1
\end{array}\right], \tau_{i}^{c}=\left[\begin{array}{l}
0 \\
0 \\
0
\end{array}\right], i=1,2, \cdots, 5 .
$$

The homogeneous transformation matrix of the local coordinate system relative to the global coordinate system is:

$$
T_{B i}=\left[\begin{array}{cc}
R_{B i} & p_{B i} \\
0 & 1
\end{array}\right], p_{B i}=r_{B i}, i=1,2, \cdots, 5
$$

The expression of wrench in the global coordinate system is:

$$
\begin{aligned}
& F_{i}^{B}=A^{\mathrm{T}}\left(T_{B i}^{-1}\right) F_{i}^{C}, A^{\mathrm{T}}\left(T_{B i}^{-1}\right)=\left[\begin{array}{cc}
R_{B i} & 0 \\
\hat{p}_{B i} R_{B i} & R_{B i}
\end{array}\right], \\
& F_{i}^{B}=f_{i}\left[\begin{array}{l}
n_{i} \\
r_{i} \times n_{i}
\end{array}\right]=\left[\begin{array}{l}
f_{i}^{B} \\
\tau_{i}^{B}
\end{array}\right] .
\end{aligned}
$$

In this way, the total wrench in the global coordinate system can be obtained as:

$$
F^{B}=\sum_{i=1}^{5} F_{i}^{B}=\sum_{i=1}^{5} f_{i}\left[\begin{array}{l}
n_{i} \\
r_{i} \times n_{i}
\end{array}\right], i=1,2, \cdots, 5 .
$$

The consequence of simulation is placed in Figure 5(b), the abscissa is the diameter of the virtual ball, and the ordinate is the resultant force received by the virtual ball. We can see that the sphere's resultant force in a heterogeneous hand is always less than in a homogeneous hand. Less resultant force means that the object is more easily balanced by the friction of fingers and balanced grab state is easier to achieve. This simulation proved that the force distribution of human fingers can reach a better effect in grasping objectives.

For a better imitation to human hand and a better crawling effect, relative strong thumb, index finger, and middle finger are needed, so BEPT1 was selected to these 3 fingers in the humanoid hand to produce a large fingertip force. In regard to ring finger and little finger, we selected BEPT4 to the ring finger and BEPT5 to little finger for an auxiliary grasping. The corresponding BEPT selection can be seen in Table 2 .

\section{Humanoid Hand Manufacture and Experiments} 4.1 Humanoid Hand Manufacture

A DLP 3D-printer is applied in the manufacturing process of humanoid for a fast forming. The palm and

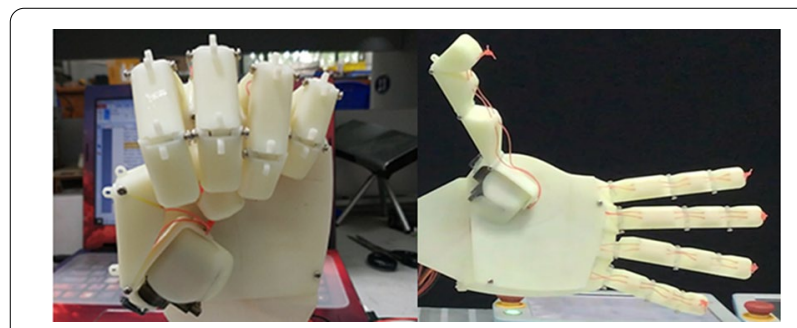

Figure 6 Manufactured humanoid hand 
fingers' rigid parts are 3D-printed through common photosensitive resin, and the peripheral flexible fence is manufactured by industrial flexible polyurethane.

The manufactured humanoid hand is shown in Figure 6. Due to the small, light, and effective transmission of BEPT, a great grasping performance can be satisfied with this hand. The price of humanoid hand is less than 50 dollars with an equal size to human hand. The humanoid hand's largest fingertip grasping force can reach $33 \mathrm{~N}$ and fastest fingertip speed is $0.6 \mathrm{~s} / 180^{\circ}$, which are almost equal to humans. After satisfying the above conditions, the weight of the 3D-printed humanoid hand is controlled below $430 \mathrm{~g}$, which is basically equal to the weight of an adult man's hand (approximately 400-500 g).

\subsection{Single Finger Experiment}

In this section, a single finger grasping experiment was conducted to verify our previous simulation. Before experiments, the control strategy of fingers should be analyzed.

Based on previous research, the operating modes of BEPT can be obtained. According to the contact process between finger and object, the operating modes of BEPT can be separated as:

1) Fast mode, fast mode takes place when the finger does not contact with the object. In this time, the force of fingertip is small, and so is the cable tension, therefore the peripheral flexible fence almost is not stretched, and the transmission radius is nearly equal to $R_{o}$. So a fast fingertip speed and small fingertip force are acquired.

2) Strong mode, if the given torque of motor is large enough and a strong object the finger is encountered, the flexible fence will be fully stretched. In this time, the transmission radius is closed to $R_{i}$, a large fingertip force and small fingertip speed are acquired.
The fingers and BEPTs are driven by micro-motor, while the micro-motors' inputting voltage $U$ is constant and $I_{S}$ is inputting current. When the fingers hold the object tightly, micro-motor will reach a stall state. In this moment, the relationship between the micro-motor largest stall torque $T_{S}$ and inputting current $I_{S}$ :

$$
T_{S}=K_{t} \Phi I_{S} .
$$

So we can get that $T_{S} i s$ a function of current $I_{S}$. We can control the cable tension $T$ through control the inputting current $I_{S}$. If a small current is inputted, the BEPT will do a passive force compliance according to the small micromotor torque. If inputted current is big enough, the BEPT will contract completely, the transmission radius is closed to $R_{i}$. In this time the largest fingertip force is decided by the micro-motor's stall torque $T_{S}$.

Experimental setup is shown in Figure 7(a). A digital power produced a standard voltage of $12 \mathrm{~V}$ to micro motor, micro motor drove the BEPT then the finger press on ATI sensor. The ATI sensor recorded the forces and Torques of three axes and sends them to the $\mathrm{PC}$, which is responsible for data processing and visualization. In the experiment, we maintained the voltage of the micro motor at $12 \mathrm{~V}$ and gradually increased the current of the motor from $0.1 \mathrm{~A}$ to $0.7 \mathrm{~A}$. In each case, we recorded the fingertip pressure on the ATI sensor, and the experiment was performed five times in each case.

The green solid curve in Figure 7(b) is a typical fingertip force change when the current is equal to 0.7 A. The red dotted line is the force change curve when grabbing a rigid objective in simulation. We can see that the result of the experiment is very similar to the simulation, in the first stage, BEPT does not fully contract, and the force increases relatively slowly. In this stage, BEPT is doing a passive force compliance. In the

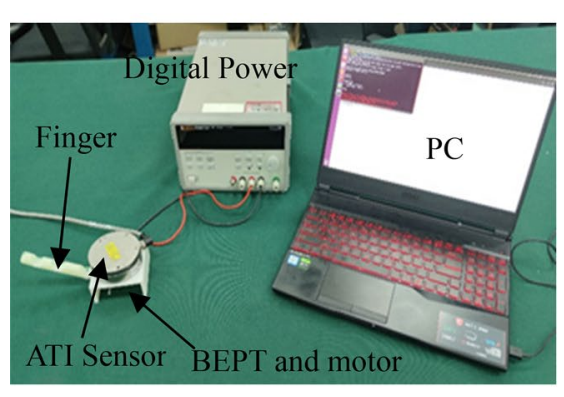

(a)

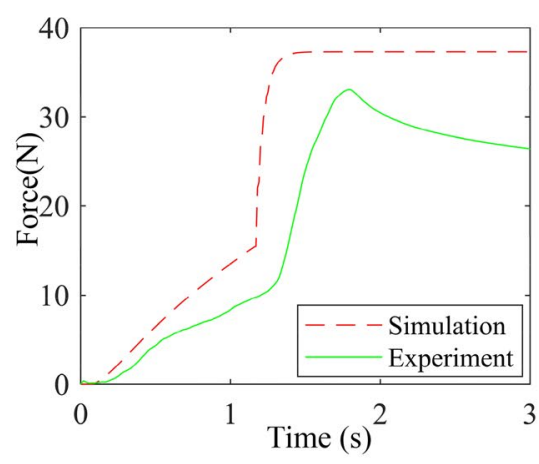

(b)

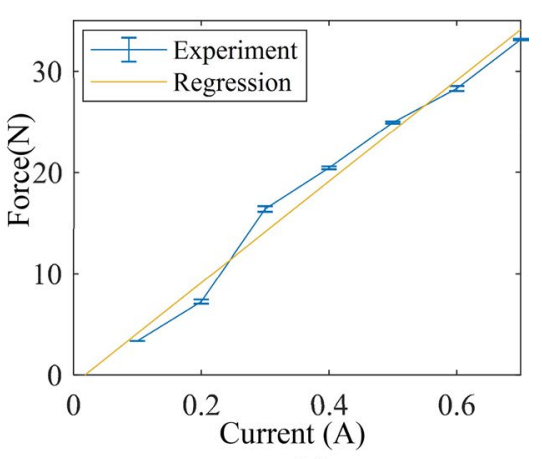

(c)

Figure 7 (a) Single finger experiment setting, (b) Comparison of fingertip force curve and simulation, (c) The relationship between fingertip force and current fingertip 
second stage, BEPT completely contracts, the motor stalls, the force increases rapidly and the finally fingertip force is determined by the current.

At the same time, we recorded the relationship between the fingertip force and the current. The force of the fingertip is the average of five experiments. It can be seen in Figure 7(c) that the fingertip force presents a good linear relationship with the current, and the variance of the fingertip force is relatively small under a specific current. The blue line represents the experimental data and the yellow line represents the currentforce curve after regression. Experiments proved that we can perform a preliminary fingertip force control by controlling the current of the motor.

\subsection{Humanoid Hand Grasping Experiment}

Based on previous research, we know that we can control fingertip force by controlling the current input to the motor, and the humanoid hand we designed has a force distribution similar to human being. To verify the outstanding grasping performance of designed humanoid hand, two kinds of experiments are conducted, grasping force compliance experiment and grasping diversity test.

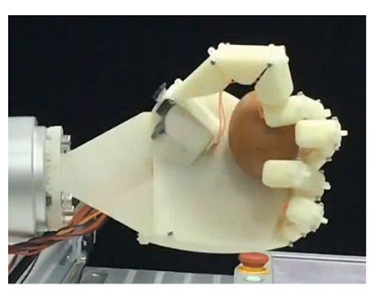

(a)

(c)

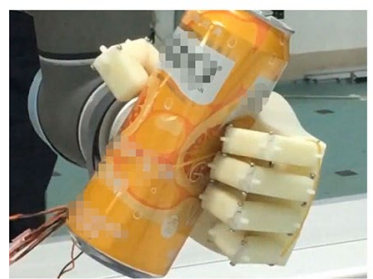

(e)

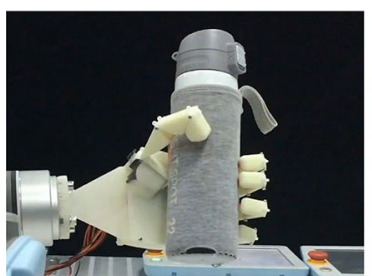

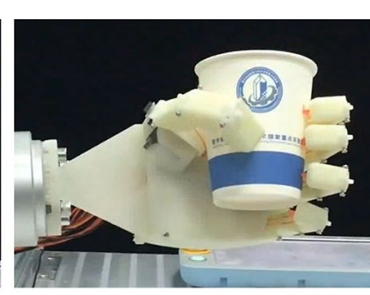

(b)

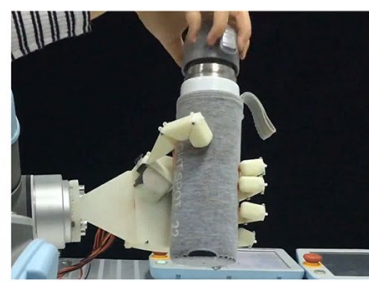

(d)

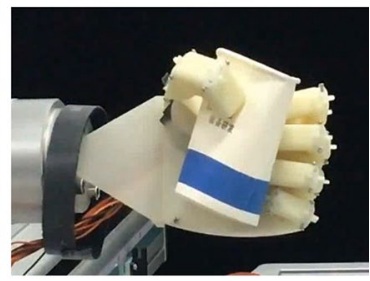

(f)
Figure 8 (a) Grasp a raw egg, (b) Hold a paper cup, (c, d) Open the thermos lid, (e) Damage a metal can, (f) Damage a paper cup

\subsubsection{Grasping Force Compliance Experiment}

Benefit from the advantages of BEPT, a good force compliance performance is obtained. A force compliance experiment is implemented in order to exam the good force compliance performance of the manufactured humanoid hand.

In this experiment, an open-loop control strategy is applied to make BEPT do a passive force compliance. In a condition that micro-motors' inputting voltage $12 \mathrm{~V}$ and current $0.1 \mathrm{~A}$, the humanoid hand is required to grasp a raw egg and an empty paper cup. Then we adjusted the micro-motors' inputting voltage to $12 \mathrm{~V}$ and current at 0.7 A. A metal can, a paper cup and a thermos cup are grasped by manufactured humanoid hand.

Through Figure 8(a) and (b), we can know that the raw eggs were not crushed, and the empty paper cups were not deformed. When we increase the current, the hand can easily damage the paper cup and metal can. It can also grip the thermos cup tightly that the thermos lid could be opened by another human hand. Through force compliance experiments, it can be proved that we can initially control the grasping force of the prosthetic hand by controlling the input current of the motor.

\subsubsection{Grasping Diversity Test}

Based on BEPT, we designed our prosthetic hand. In order to evaluate the grasping ability of the prosthetic hand and more comprehensively evaluate the grasping ability of the manipulator, we choose GRASP motion spectrum [30] as the reference for the grasping

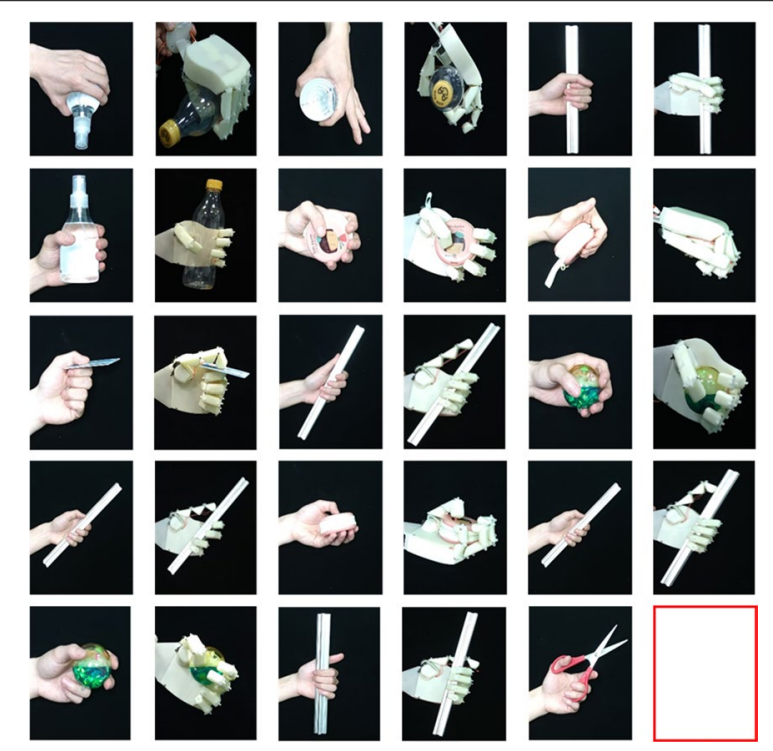

Figure 9 Comparison of prosthetic hand and human hand in motion spectrum 
behavior of the prosthetic hand. There are 33 grasping modes in the motion spectrum, covering the most common movements of human hands, of which 15 are covered grasping.

We use 15 kinds of hand movement modes of covered grasping as a reference, and do not consider some very delicate operations of the hand. After that, we let the humanoid hand grasp the corresponding object imitating the human grabbing mode. The results of the experiment showed in Figure 9 proved that our prosthetic hands can complete 14 of them. For operations such as holding scissors, the fingers are required to have the ability to extend horizontally. We did not consider this degree of freedom when designing. However, our manipulator can still complete most of the motion modes of human hand covering and grasping, showing a good grasping ability.

\section{Conclusions}

In this paper, a 3D-printed cable driven humanoid hand based on BEPT is designed. A DLP 3D-printer is applied to manufacture various parts of humanoid hand. The kinematic and dynamic behavior of the prosthetic hand fingers are analyzed. At the same time, in order to have a better bionic effect and better grasping performance, we selected specific BEPT for specific finger based on the mechanical properties of corresponding human finger.

The manufactured humanoid hand can reach a great balance between the price and various kinds of humanoid hand. With a price less than 50 dollars and equal size to human hand, the humanoid hand has a $33 \mathrm{~N}$ largest fingertip grasping force and $0.6 \mathrm{~s} / 180^{\circ}$ fastest fingertip speed. After satisfying the above conditions, the weight of 3D-printed humanoid hand is less than $430 \mathrm{~g}$ (adult man: $400-500 \mathrm{~g}$ ). The results of the experiments verified the correctness of our simulation, showed that a great performance is reached through BEPT. It has achieved great results in terms of price and weight, gripping force, gripping speed, and force control effects.

However, our research also has some shortcomings. For example, our finger's under-actuation implementation scheme does not decouple the degrees of freedom of the finger, resulting in some more sophisticated operations that cannot be completed. Flexible transmission mechanism can also cause some material fatigue problems. Experiments on the dexterity of prosthetic hands are also not perfect. These are the directions for our future research. This prosthetic hand has a broad application field like artificial prosthesis, humanoid robot and Human-Robot interaction.

Acknowledgements

Not applicable.

\section{Authors' Information}

Teru Chen, received his B.S degree from Huazhong University of Science and Technology (HUST), China, in 2019. He is currently working toward the Ph.D. degree at State Key Laboratory of Digital Manufacturing Equipment and Technology, Huazhong University of Science and Technology (HUST), China. His research interests mainly include advanced mechanism design and surgical robot technology.

Xingwei Zhao, received B.S. and M.S. degrees in mechanical engineering from University of Duisburg-Essen, Duisburg, Germany, in 2012 and 2013, respectively. He received the Ph.D. degree in mechanical engineering from the Technical University of Berlin, Berlin, Germany, in 2017. He is currently an associate researcher with the State Key Laboratory of Digital Manufacturing Equipment and Technology, Huazhong University of Science and Technology (HUST), China. His research interests mainly include nonlinear dynamics, nonlinear control and robotic manufacture.

Guocai Ma, born in 1989, received B.S. and PhD of Engineering from Beihang University, China, in 2012 and 2019, respectively. He is currently working at State Key Laboratory of Intelligent Manufacturing System Technology, Beijing Institute of Electronic System Engineering, China. His research interests include robotic application technology, additive manufacturing, and intelligent assembly technology.

Bo Tao, received the B.S. and PhD degrees in mechanical engineering from Huazhong University of Science and Technology (HUST), China, in 1999 and 2007 respectively. From 2007 to 2009, he was a post-doctor at the Department of Electronics Science and Technology, HUST, China. After that, he has been an Associate Professor in 2009 and Professor in 2013 at School of Mechanical Science and Engineering, HUST, China. He has published more than 40 papers in international journals. His research interests include intelligent manufacturing and robotics technologies, RFID technologies and applications.

Zhouping Yin, received the B.S., M.S. and PhD degrees in mechanical engineering from Huazhong University of Science and Technology (HUST), China, in 1994, 1996 and 2000, respectively. He is a professor at School of Mechanical Science and Engineering, HUST, China. Since 2005, he has been Vice Head of the State Key Laboratory of Digital Manufacturing Equipment and Technology, HUST, China. He was awarded the China National Funds for Distinguished Young Scientists in 2006. He is a "Cheung Kong" Chair Professor of HUST since 2009. His research interests include electronic manufacturing equipment and technology, RFID technology and applications, digital manufacturing and applications.

\section{Authors' Contributions}

$X Z$ and GM was in charge of the whole trial; TC wrote the manuscript; BT and $Z Y$ directed the writing of the papers. All authors read and approved the final manuscript.

\section{Funding}

Supported by National Natural Science Foundation of China (Grant No. 91948301), Hubei Provincial Technology Innovation Project of China (Grant No. 2019AAA071), and Open Fund of State Key Laboratory of Robotics and System (Grant No. SKLRS-2019-KF-11).

\section{Availability of Data and Materials}

The datasets supporting the conclusions of this article are included within the article.

\section{Competing Interests}

The authors declare no competing financial interests.

\section{Author Details}

${ }^{1}$ State Key Laboratory of Digital Manufacturing Equipment and Technology, Huazhong University of Science and Technology, Wuhan 430074, China. ${ }^{2}$ State Key Laboratory of Intelligent Manufacturing System Technology, Beijing Institute of Electronic System Engineering, Beijing 100854, China.

Received: 15 August 2020 Revised: 28 March 2021 Accepted: 5 August 2021

Published online: 21 August 2021 


\section{References}

[1] S Almecija, J B Smaers, W L Jungers. The evolution of human and ape hand proportions. Nature Communications, 2015, 6

[2] M J Liu, C H Xiong, D Hu. Assessing the manipulative potentials of monkeys, apes and humans from hand proportions: implications for hand evolution. Proceedings of the Royal Society B-Biological Sciences, 2016, 283(1843).

[3] D Hu, C H Xiong, M J Liu. Exploring the existence of better hands for manipulation than the human hand based on hand proportions. Journal of Theoretical Biology, 2018, 440: 100-111.

[4] L Göran. The hand and the brain: from Lucy's thumb to the thought-controlled robotic hand. New York: Springer, 2013.

[5] J Guo, X He, C Xiong. Simultaneous motion and force sensing for a flexure finger. 2020 8th IEEE RAS/EMBS International Conference for Biomedical Robotics and Biomechatronics (BioRob), 29 Nov.-1 Dec., 2020: 1061-1066.

[6] G Carbone. Grasping in robotics. New York: Springer, 2013.

[7] J T Li, S Wang, J Wang, et al. Development of a hand exoskeleton system for index finger rehabilitation. Chinese Journal of Mechanical Engineering, 2012, 25(2): 223-233.

[8] C Pylatiuk, S Mounier, A Kargov, et al. Progress in the development of a multifunctional hand prosthesis. Proceedings of the 26th Annual International Conference of the IEEE Engineering in Medicine and Biology Society, 2004, 26: 4260-4263.

[9] M C Carrozza, G Cappiello, S Micera, et al. Design of a cybernetic hand for perception and action. Biological Cybernetics, 2006, 95(6): 629-644.

[10] J T Belter, A M Dollar. Performance characteristics of anthropomorphic prosthetic hands. 2011 IEEE International Conference on Rehabilitation Robotics (ICORR), Zurich, Switzerland, 2011: 1-7.

[11] C Pylatiuk, S Schulz, L Doderlein. Results of an Internet survey of myoelectric prosthetic hand users. Prosthetics and Orthotics International, 2007, 31(4): 362-370.

[12] Li Jiang, Hong Liu, Zongwu Xie, et al. Design of a novel dexterous robot hand. Chinese Journal of Mechanical Engineering, 2004, 17(3): 360-363.

[13] W C Lee, C W Wu. A novel design of a prosthetic hand. IEEE International Conference on Systems, Man and Cybernetics, 2010.

[14] P Wattanasiri, P Tangpornprasert, C Virulsri. Design of multi-grip patterns prosthetic hand with single actuator. IEEE Transactions on Neural Systems and Rehabilitation Engineering, 2018, 26(6): 1188-1198.

[15] J Ingvast, J Wikander, C Ridderstrom. The PVT, an elastic conservative transmission. International Journal of Robotics Research, 2006, 25(10): 1013-1032.

[16] Y J Shin, H J Lee, K S Kim, et al. A robot finger design using a dual-mode twisting mechanism to achieve high-speed motion and large grasping force. IEEE Transactions on Robotics, 2012, 28(6): 1398-1405.
[17] S M Felton, D Y Lee, K J Cho, et al. A passive, Origami-inspired, continuously variable transmission. 2014 IEEE International Conference on Robotics and Automation (ICRA), Hong Kong, China, 2014: 2913-2918.

[18] S L Qiao, R Q Liu, HW Guo, et al. Configuration design of an underactuated robotic hand based on maximum grasping space. Chinese Journal of Mechanical Engineering, 2018, 31: 35, https://doi.org/10.1186/ s10033-018-0243-4

[19] J H Geng, H A Qiang, W M Zhang, et al. Design of a humanoid hand with 5 fingers and 14 joints driven by 2 motors. Eleventh World Congress in Mechanism and Machine Science, Tianjin, China, 2004: 2003-2006.

[20] Q Zhang, T Pi, R Liu, et al. Simultaneous and proportional estimation of multijoint kinematics from EMG signals for Myocontrol of robotic hands. IEEE/ASME Transactions on Mechatronics, 2020, 25(4): 1953-1960.

[21] C H Xiong, W R Chen, B Y Sun, et al. Design and implementation of an anthropomorphic hand for replicating human grasping functions. IEEE Transactions on Robotics, 2016, 32(3): 652-671.

[22] W R Chen, C H Xiong, M J Liu, et al. Characteristics analysis and mechanical implementation of human finger movements. 2014 IEEE International Conference on Robotics and Automation (ICRA), 2014: 403-408.

[23] T Duan, J Huang, Z Xie, et al. Continuous control of wrist-hand prosthesis by extracting independents EMG signals from cross-talk muscle groups. 2019 Chinese Control Conference (CCC), 27-30 July, 2019: 4537-4542.

[24] X Yong, X B Jing, Y L Jiang, et al. Tendon drive finger mechanisms for an EMG prosthetic hand with two motors. 2014 7th International Conference on Biomedical Engineering and Informatics (BMEl 2014), Dalian, China, 2014 568-572.

[25] L C Lei, H P Liu, F C Sun, et al. The intelligent grasping tactics of dexterous hand. 2014 13th International Conference on Control Automation Robotics \& Vision (ICARCV), 2014: 352-357.

[26] S A Dalley, TE Wiste, T J Withrow, et al. Design of a multifunctional anthropomorphic prosthetic hand with extrinsic actuation. IEEE-ASME Transactions on Mechatronics, 2009, 14(6): 699-706.

[27] V Bundhoo, E J Park. Design of an artificial muscle actuated finger towards biomimetic prosthetic hands. 2005 12th International Conference on Advanced Robotics, 2005: 368-375.

[28] KW O'Brien, P A Xu, D J Levine, et al. Elastomeric passive transmission for autonomous force-velocity adaptation applied to 3D-printed prosthetics. Science Robotics, 2018, 3(23): eaau5543.

[29] C Mehring, M Akselrod, L Bashford, et al. Augmented manipulation ability in humans with six-fingered hands. Nature Communications, 2019, 10(1).

[30] I M Bullock, J Z Zheng, S De La Rosa, et al. Grasp frequency and usage in daily household and machine shop tasks. IEEE Transactions on Haptics, 2013, 6(3): 296-308

\section{Submit your manuscript to a SpringerOpen ${ }^{\circ}$ journal and benefit from:}

- Convenient online submission

- Rigorous peer review

- Open access: articles freely available online

- High visibility within the field

- Retaining the copyright to your article

Submit your next manuscript at $\boldsymbol{\nabla}$ springeropen.com 